\title{
Multiple morphogenic culture systems cause loss of resistance to cassava mosaic disease
}

\author{
Raj Deepika Chauhan, Getu Beyene and Nigel J. Taylor*
}

\begin{abstract}
Background: Morphogenic culture systems are central to crop improvement programs that utilize transgenic and genome editing technologies. We previously reported that CMD2-type cassava (Manihot esculenta) cultivars lose resistance to cassava mosaic disease (CMD) when passed through somatic embryogenesis. As a result, these plants cannot be developed as products for deployment where CMD is endemic such as sub-Saharan Africa or the Indian sub-continent.

Result: In order to increase understanding of this phenomenon, 21 African cassava cultivars were screened for resistance to $C M D$ after regeneration through somatic embryogenesis. Fifteen cultivars were shown to retain resistance to CMD through somatic embryogenesis, confirming that the existing transformation and gene editing systems can be employed in these genetic backgrounds without compromising resistance to geminivirus infection. CMD2-type cultivars were also subjected to plant regeneration via caulogenesis and meristem tip culture, resulting in $25-36 \%$ and $5-10 \%$ of regenerated plant lines losing resistance to CMD respectively.
\end{abstract}

Conclusions: This study provides clear evidence that multiple morphogenic systems can result in loss of resistance to $C M D$, and that somatic embryogenesis per se is not the underlying cause of this phenomenon. The information described here is critical for interpreting genomic, transcriptomic and epigenomic datasets aimed at understanding CMD resistance mechanisms in cassava.

Keywords: Cassava, Cassava mosaic disease, Meristem tip culture, Organogenesis, Somatic embryogenesis

\section{Background}

Cassava mosaic disease (CMD) is endemic throughout Sub-Saharan Africa and the Indian sub-continent. Effective resistance to the whitefly-vectored geminiviruses that cause CMD is essential to secure yields for cassava farmers across these regions. Three genetic sources of CMD resistance, i.e. CMD1, CMD2 and CMD3, have been identified. CMD1 resistance was introgressed from Manihot glaziovii and understood to be multigenic and recessive, while CMD2 is monolocus, dominant in nature and was identified in landraces collected in Nigeria and Benin/Togo [1, 2]. CMD3 carries the CMD2 locus plus an additional QTL [3]. In all three resistance types, the underlying genes and molecular mechanisms remain unknown. We recently reported that all plants of

\footnotetext{
* Correspondence: ntaylor@danforthcenter.org

Donald Danforth Plant Science Center, St. Louis, MO, USA
}

CMD2-type cultivars regenerated through somatic embryogenesis lose resistance to CMD and develop severe mosaic symptoms when inoculated with infectious geminivirus clones in the greenhouse, and when exposed to viliferous whiteflies in the field. Cultivars tested that carry CMD1 and CMD3 resistance mechanisms did not suffer from this phenomenon with plants regenerated through somatic embryogenesis remaining resistant to CMD [4].

Uniform and consistent loss of a major trait such as virus resistance in multiple cultivars by simple passage through embryogenesis is unique in the literature. Increasing understanding of why CMD2 resistance is compromised in this manner is imperative to the success of cassava enhancement programs. In general, phenotypic variations in plants recovered through tissue culture can be attributed to genetic or epigenetic changes. Changes in the DNA methylation status of the cassava genome

(c) The Author(s). 2018 Open Access This article is distributed under the terms of the Creative Commons Attribution 4.0 International License (http://creativecommons.org/licenses/by/4.0/), which permits unrestricted use, distribution, and 
were reported in plants regenerated via meristem tip culture by Kitimu et al. [5].

The single locus, dominant nature of CMD2 makes it highly favored by breeders as a source of resistance to generate improved planting materials [6]. Reliance on a single gene mechanism, however, risks evolution of the pathogen to overcome the resistance. Indeed, breakdown of CMD2-mediated resistance was reported recently under greenhouse conditions by Ndunguru, et al. [7]. Advanced biotechnologies in cassava rely on induction of somatic embryogenesis to generate the totipotent tissues utilized for transgene integration and delivery of gene editing reagents $[8,9]$. Genetic modification in this manner must be achieved without losing resistance to CMD, a trait that is essential in all enhanced cassava germplasm intended for deployment in Africa and India.

We report here further evidence for loss of functional CMD2-mediated resistance when tissues are passed through morphogenic culture systems. In addition to somatic embryogenesis, information is presented describing the effects of caulogenesis and meristem tip culture on loss of resistance to $\mathrm{CMD}$ in regenerated plants.

\section{Methods}

\section{Media composition and culture conditions}

Compositions of culture media used in this study followed Chauhan, et al. [10] for induction of organized embryogenic structures (OES) and friable embryogenic callus (FEC); Chauhan and Taylor [11] for organogenesis; and the International Institute of Tropical Agriculture Handbook [12] for meristem tip culture. Media components, antibiotics, growth regulators and additives were procured from Sigma (St. Louis, MO, USA). Meta-topolin $(m \mathrm{~T})$ used for regeneration of plants through organogenesis was obtained from Duchefa Biochemie, The Netherlands. All in vitro cultures were incubated at $28 \pm 1^{\circ} \mathrm{C}$ with $16 \mathrm{~h}$ light/ $8 \mathrm{~h}$ dark photoperiod under fluorescent lamps at $75 \mu \mathrm{mol} \mathrm{m}{ }^{-2} \mathrm{~s}^{-1}$ unless otherwise specified.

\section{Plant material and gene constructs}

In vitro shoot cultures of CMD1-type cassava cultivar TMS 30572, CMD2-type cultivars TME 419, TME B7, CMD3-type cultivars TMS 98/0581, TMS 98/0505, TMS 96/1632 and other cultivars with unknown CMD-types NR 03/0155, TMS 98/0002, TMS 01/0040, TMS 92/ 0057, TMS 01/1206, TMS 91/02324, TMS 98/2132, TMS 92/0326, TMS 01/1371, TMS 95/0289 and 60444 were obtained from IITA, Nigeria (Table 1). Stem cuttings of CMD1-type cultivars NASE 3, NASE 14 and CMD2-type cultivars TME 14, TME 204 were imported from the National Crops Resources Research Institute (NaCRRI), Uganda, and TME 7 from IITA collected from farmer fields in Nigeria. Stem cuttings were established under in vitro conditions at the Donald Danforth
Plant Science Center (DDPSC), St. Louis, MO, USA. Axillary buds that developed from the stems were excised and established in tissue culture following methods described by Taylor, et al. [13] and Chauhan, et al. [10]. CMD susceptible plants of TME 204 were obtained by regeneration from friable embryogenic callus (FEC-TME 204) [10]; [4] and served as known negative controls for greenhouse trials.

Agrobacterium tumefaciens strain LBA4404 harboring a pCAMBIA2300-based binary vector containing the enhanced green fluorescent protein gene $(e g f p)$ under control of the Cauliflower mosaic virus (CaMV) 35S promoter was used for transformation experiments, following procedures described by Chauhan, et al. [10].

\section{Production of organized embryogenic structures (OES) and plant regeneration}

Induction of organized embryogenic structures (OES) was performed as described by Taylor, et al. [13] and Chauhan, et al. [10]. Immature leaf lobe explants were excised from 4- to 6-week-old micropropagated shoot cultures and placed on DKW/Juglans basal salts [14] (PhytoTechnology Laboratories, Kansas, USA) plus Murashige and Skoog (MS) [15] vitamins, supplemented with $2 \% w / v$ sucrose and $50 \mu \mathrm{M}$ picloram (DKW 50P). Cultures were incubated in the dark at $28{ }^{\circ} \mathrm{C}$ for 4 weeks. Eight leaf lobe explants were cultured per plate with five plates per cultivar, and experiments replicated three times. The number of explants forming OES was assessed 5 weeks after explanting.

Plants were regenerated 8-10 weeks after leaf lobe explant initiation by excising OES from the non-embryogenic tissues and subculture onto MS media containing $2 \%$ sucrose $w / v$ (MS2) and $2 \mu \mathrm{M} m \mathrm{~T}$ solidified with $0.22 \% w / v$ gelzan [11]. Between eight and 10 colonies of OES were cultured in each plate. After 4 weeks, individual cotyledon stage embryos were separated from each other and subcultured onto fresh media of the same type. Germinating shoots possessing two to three true leaves were transferred for rooting to MS media supplemented with $2 \% w / v$ sucrose and solidified with $0.8 \% w /$ $v$ Noble agar.

\section{Agrobacterium-mediated transformation and plant regeneration}

Friable embryogenic callus (FEC) produced from six cultivars TMS 98/0505, TMS 01/0040, TMS 01/1206, TMS 91/02324, TME B7 and TME 419 was transformed with Agrobacterium tumefaciens strain LBA4404 harboring a pCAMBIA2300-based binary vector carrying egfp following the method described by Chauhan, et al. [10]. Agrobacterium suspension at an $\mathrm{OD}_{600}$ of 0.05 was used to inoculate FEC and the cultures were kept at $22{ }^{\circ} \mathrm{C}$ under constant light. Three to 4 days after the inoculation, the 
Table 1 Induction of organized embryogenic structures (OES), friable embryogenic structures (FEC) from cassava cultivars and response to MeSPY1-VIGS cassava mosaic disease challenge

\begin{tabular}{|c|c|c|c|c|c|c|c|}
\hline \multirow[t]{2}{*}{ Cultivar name } & \multirow[t]{2}{*}{$\begin{array}{l}\text { Resistance } \\
\text { type }\end{array}$} & \multirow{2}{*}{$\begin{array}{l}\text { Organized } \\
\text { embryogenic } \\
\text { structures (OES) } \\
\text { induction } \\
\text { frequency (\%) }\end{array}$} & \multirow{2}{*}{$\begin{array}{l}\text { Friable } \\
\text { Embryo-genic } \\
\text { Callus (FEC) } \\
\text { induction (Yes/No) }\end{array}$} & \multicolumn{2}{|c|}{$\begin{array}{l}\text { Number of dead plants/total } \\
\text { plants challenged with MeSPY1-VIGS }\end{array}$} & \multicolumn{2}{|c|}{$\begin{array}{l}\text { Resistance/ susceptibility } \\
\text { to cassava mosaic disease }\end{array}$} \\
\hline & & & & Wildtype & OES-derived & Wildtype & OES-derived \\
\hline NASE 3 & CMD1 & 24 & No & $1 / 8$ & $3 / 8$ & Resistant & Resistant \\
\hline NASE 14 & CMD1 & 81 & Yes & $0 / 9$ & $0 / 10$ & Resistant & Resistant \\
\hline TMS 30572 & CMD1 & 28 & No & $2 / 9$ & $1 / 8$ & Resistant & Resistant \\
\hline TME 204 & CMD2 & 81 & Yes & $1 / 9$ & $7 / 7^{a}$ & Resistant & Susceptible \\
\hline TME B7 & CMD2 & 95 & Yes & $1 / 9$ & $6 / 6$ & Resistant & Susceptible \\
\hline TME 419 & CMD2 & 58 & Yes & $7 / 9$ & $6 / 6$ & Susceptible & Susceptible \\
\hline TMS 96/1632 & CMD3 & 63 & No & $0 / 6$ & $0 / 9$ & Resistant & Resistant \\
\hline TMS 98/0505 & CMD3 & 55 & Yes & $0 / 8$ & 0/9 & Resistant & Resistant \\
\hline TMS 98/0581 & CMD3 & 66 & No & $0 / 7$ & $0 / 10$ & Resistant & Resistant \\
\hline TMS 92/0326 & Unknown & 89 & Yes & $1 / 8$ & $1 / 7$ & Resistant & Resistant \\
\hline TMS 92/0057 & Unknown & 76 & No & $0 / 12$ & $0 / 5$ & Resistant & Resistant \\
\hline TMS 95/0289 & Unknown & 3 & No & $3 / 11$ & NA & Resistant & Not tested \\
\hline TMS 98/2132 & Unknown & 79 & No & $0 / 6$ & $0 / 4$ & Resistant & Resistant \\
\hline NR03/0155 & Unknown & 53 & No & 0/9 & $0 / 8$ & Resistant & Resistant \\
\hline TMS 91/02324 & Unknown & 53 & Yes & $0 / 10$ & 0/9 & Resistant & Resistant \\
\hline TMS 98/0002 & Unknown & 78 & No & $0 / 10$ & $0 / 10$ & Resistant & Resistant \\
\hline TMS 01/0040 & Unknown & 59 & Yes & $0 / 10$ & 0/9 & Resistant & Resistant \\
\hline TMS 01/1206 & Unknown & 66 & Yes & $0 / 7$ & $0 / 8$ & Resistant & Resistant \\
\hline TMS 01/1371 & Unknown & 64 & No & $0 / 8$ & 0/9 & Resistant & Resistant \\
\hline Mbundamali & Unknown & Not determined & Not tested & $10 / 10$ & $5 / 5$ & Susceptible & Susceptible \\
\hline 60444 & Susceptible & 90 & Yes & $9 / 9$ & NA & Susceptible & Not tested \\
\hline
\end{tabular}

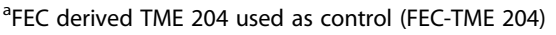

Agrobacterium was washed off from the FEC. The tissues were then selected on media containing $27.5 \mu \mathrm{M}$ paromomycin followed by transfer to embryo maturation media containing $45 \mu \mathrm{M}$ paromomycin. The cotyledon stage embryos were germinated and rooted on selection free media. GFP-expressing tissues were visualized under a Nikon C15304 dissecting microscope equipped with an excitation filter of 460-500 $\mathrm{nm}$ and barrier filter, $510 \mathrm{LP}$ at different stages after transformation and scored as described by Chauhan, et al. [10]. Three replicates were established per cultivar for each treatment and transformation experiments repeated two times. Non-transgenic plants for use as negative controls were recovered from non-transformed FEC.

\section{Regeneration of cassava plants through organogenesis} Plants of CMD2-type cultivars TME 7 and TME 204 were regenerated from leaf-petiole explants following Chauhan and Taylor [11]. Leaf-petiole explants were excised from mother plants pre-treated with $2 \mu \mathrm{M} m \mathrm{~T}$ for 4 weeks, cultured on MS medium supplemented with
$2 \% w / v$ sucrose, $1 \mu \mathrm{M} 2,4-\mathrm{D}$ and $1 \mu \mathrm{M} m \mathrm{~T}$ for 7 days, followed by transfer to MS2 medium containing $6 \mu \mathrm{M}$ $m \mathrm{~T}$. Tissues were subcultured onto fresh media of the same type every 2-3 weeks. Regenerated shoots 2.0 to $2.5 \mathrm{~cm}$ in length were transferred to MS2 media for rooting and plantlet establishment.

\section{Regeneration of cassava plants through meristem tip culture}

Plants of CMD2-type cultivars TME 7, TME 14, TME 204, CMD1-type cultivar TMS 30752 and CMD3-type cultivar TMS 98/0505 were regenerated through meristem tip culture following the method described by IITA [12]. Six- to eight-week-old in vitro micropropagated mother plants cultured on MS media supplemented with $2 \% \mathrm{w} / v$ sucrose (MS2) and solidified with $0.8 \% \mathrm{w} / v$ noble agar were used as the explant source. Leaf primordia were removed from the shoot tip using a hypodermic needle under a stereomicroscope (Olympus SMZ51) until the meristematic dome was visible. The meristem tip ( $0.5 \mathrm{~mm}$ in size) was excised and placed on MS 
basal media supplemented with $0.1 \mathrm{~g} / \mathrm{l}$ inositol, $0.08 \mathrm{~g} / \mathrm{l}$ adenine sulfate, $1.07 \mu \mathrm{M}$ NAA (1-napthalene acetic acid), $0.22 \mu \mathrm{M}$ BAP (6-benzylaminopurine), $0.23 \mu \mathrm{M}$ $\mathrm{GA}_{3}$ (gibberellic acid), $3 \% w / v$ sucrose and solidified with $0.4 \% w / v$ Noble agar. Cultures were incubated in the dark for two to 4 weeks at $28 \pm 1^{\circ}$ C. Regenerating shoots were rooted on MS2 media. Between 18 and 40 meristem tip explants were excised and cultured for each cultivar with the number of explants inducing shoots suitable for transferring to rooting media assessed after 5 weeks in culture.

\section{Inoculation of the plants with geminiviruses in the greenhouse}

Plants that recovered through all morphogenic pathways were propagated along with the controls on MS2 media and solidified with $0.22 \% w / v$ gelzan. After three to 4 weeks of culture, plantlets were transferred to Fafard 51 growing mixture in 3-inch pots and placed on a mist bench at $100 \%$ relative humidity for 7 days followed by transfer to the open bench at $28 \pm 1{ }^{\circ} \mathrm{C}$ day/ $25 \pm 1^{\circ} \mathrm{C}$ night temperature in a $14 \mathrm{~h}$ light/10 $\mathrm{h}$ dark photoperiod at 380 to $420 \mu \mathrm{molm}^{-2} \mathrm{~s}^{-1}$ irradiance and $80-90 \%$ relative humidity and allowed to grow for 3 weeks [13]. Plants 8 to $9 \mathrm{~cm}$ in height were transferred to a greenhouse and grown at a $32^{\circ} \mathrm{C}$ day/ $27^{\circ} \mathrm{C}$ night cycle with 70-95\% relative humidity.

A rapid VIGS-based screening method developed by Beyene et al. [16] was employed to determine the CMD status of the plants recovered from OES and meristem tip culture. Four- to six-week-old plants were inoculated with plasmid DNA of MeSPY1 (Manihot esculenta SPY) -VIGS and the DNA-B component of East African cassava mosaic virus (EACMV-K201) using a Helios ${ }^{\circ}$ Gene Gun (BioRad, Hercules, California). This causes silencing of MeSPY which leads to shoot-tip necrosis and death of the plant in CMD-susceptible cassava plants within 2-4 weeks of inoculation whereas the CMD-resistant plants remain healthy. The shoot-tip necrosis and death of plants were scored commencing 14 days after inoculation.

Plants recovered from FEC, meristem tip culture and organogenesis were inoculated with cassava geminiviruses following Beyene, et al. [4]. Four-week-old greenhouse-grown plants were inoculated with infectious clones of East African cassava mosaic virus (EACMV-K201) DNA-A GenBank: AJ717541 and DNA-B GenBank: AJ704953) [17, 18] and African cassava mosaic virus Cameroon strain (ACMV-CM) DNA-A GenBank AF112352 and DNA-B GenBank AF112353 [19] using a Helios ${ }^{\circ}$ Gene Gun (BioRad, Hercules, CA, USA). Inoculated plants were assessed for CMD symptoms starting 7 days post inoculation (DPI), with symptom severity scored on a scale of 0-5 [20] twice per week.

\section{Results}

Screening cassava cultivars for CMD resistance after passage through somatic embryogenesis

We previously reported that CMD2-type cassava plants that had been regenerated through somatic embryogenesis lose resistance to CMD but that no such effect is observed in cultivars carrying CMD1 and CMD3 resistance mechanisms [4]. To investigate this phenomenon further, 21 cassava cultivars (Table 1) from East and West Africa were passed through somatic embryogenesis by inducing OES from leaf explants [10, 13]. Plants regenerated from OES were challenged with an infectious VIGS clone of EACMV-K201 modified to carry sequences that target MeSPY1. Plants with functional resistance to geminviruses recover from this inoculation, while shoot-tip of susceptible plants wilt and die within two to 4 weeks after inoculation [16]. Plants were also inoculated with the infectious clone of EACMV- K201 [4]. Similar results were obtained from both CMD challenge methods.

All 21 cultivars tested underwent somatic embryogenesis to produce OES, with efficiencies varying from as high as $90 \%$ in 60444 , to only $3 \%$ in TMS 95/0289 (Table 1). Plants were regenerated for all cultivars (except TMS 95/0289), established in the greenhouse and subjected to inoculation with MeSPY1-VIGS. Wild-type plants of the known CMD2-types TME 204 and TME 7 demonstrated resistance to CMD and survived the MeSPY1-VIGS challenge. Conversely, shoot-tips of plants of CMD2-type cultivars regenerated from OES started to wilt 12-14 DPI and subsequently died (Fig. 1). As consistently observed in our laboratory, wild-type plants of the CMD2-type cultivar TME 419 possess low-level resistance to infection with the infectious clone EACMV-K201, although it does possess robust resistance to ACMV (data not shown). Wild-type plants of cassava cultivars Mbundamali and 60444 are CMD susceptible and remained so after regeneration through somatic embryogenesis. The remaining 15 cultivars, whether carrying CMD1, CMD3 or unknown types of resistance to CMD, remained fully resistant to inoculation with MeSPY1-VIGS after passage through somatic embryogenesis (Table 1).

FEC is the preferred target tissue for genetic transformation and is being adapted for the application of gene editing in cassava [8-10]. OES from all 21 cultivars shown in Table 1 were subcultured onto Gresshoff and Doy [21] -based medium in order to produce FEC. FEC was successfully generated from 10 cultivars, including six West African varieties that have not been reported previously (Table 1). Transgenic plant production was attempted by Agrobacterium-mediated transformation of FEC in the six cultivars TMS 98/0505, TMS 01/0040, TMS 01/1206, TMS 91/02324, TME B7 and TME 419. 

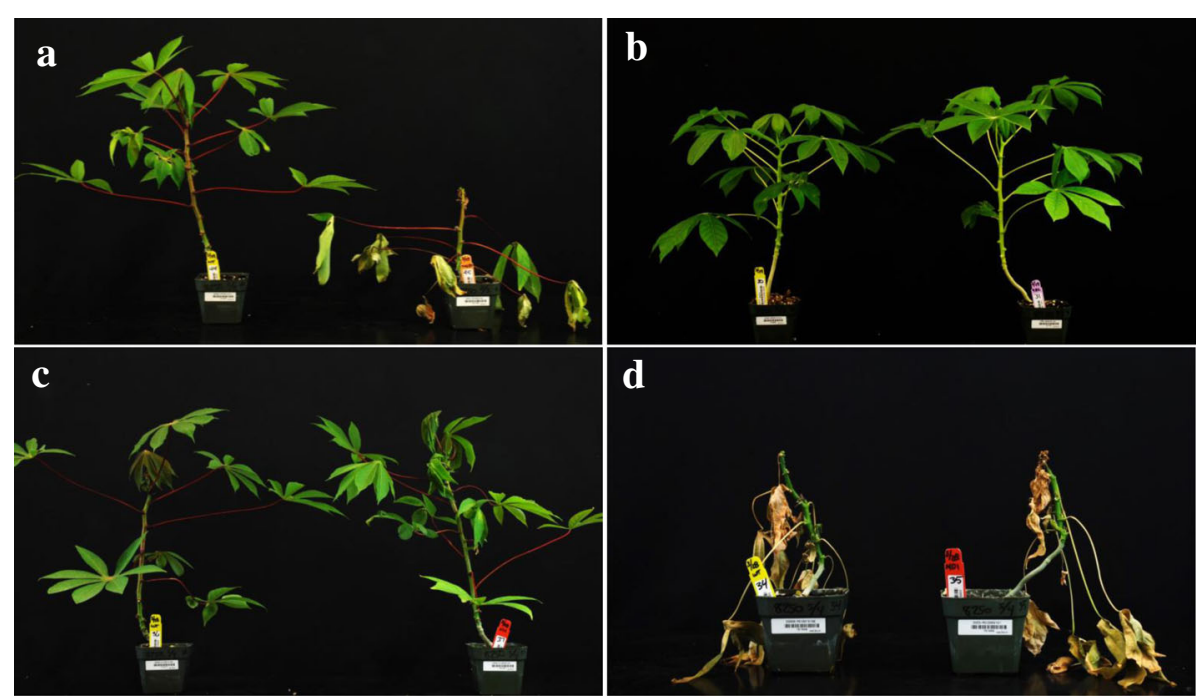

Fig. 1 Response of wild-type (left) and organized embryogenic structures (right) derived plants to inoculation with MeSPY1-VIGS to determine resistance to cassava mosaic disease. Silencing of MeSPY using MeSPY1-VIGS leads to shoot-tip necrosis and death of CMD susceptible cassava plants within 2-4 weeks after inoculation. a TME B7. b TMS 98/0002. c NASE 14. d Mbundamali

GFP-expressing callus lines were recovered in all cases (Figs. 2 and 3). As described previously [10], transformation was significantly more efficient if moxalactam was included in the culture medium prior to co-culture with Agrobacterium (Fig. 3). Transgenic plants were recovered from cultivars TMS 98/0505, TMS 01/1206 and TMS 91/02324, in addition to TME 419 and TME B7. FEC-derived plants of TMS 91/02324 (Fig. 4a \& b) and TMS 98/0505 and transgenic
GFP-expressing plant lines of TMS 98/0505 (Fig. 4c \& d) were established in the greenhouse and inoculated with EACMV-K201 (Fig. 2). Of four TMS 91/ 02324 FEC-derived, five TMS 98/0505 FEC-derived, and 24 transgenic GFP-expressing TMS 98/0505 independent lines challenged, all plants recovered to display no mosaic symptoms within five to 6 weeks after challenge (Fig. 4). This data indicates that resistance to CMD was retained through all stages of

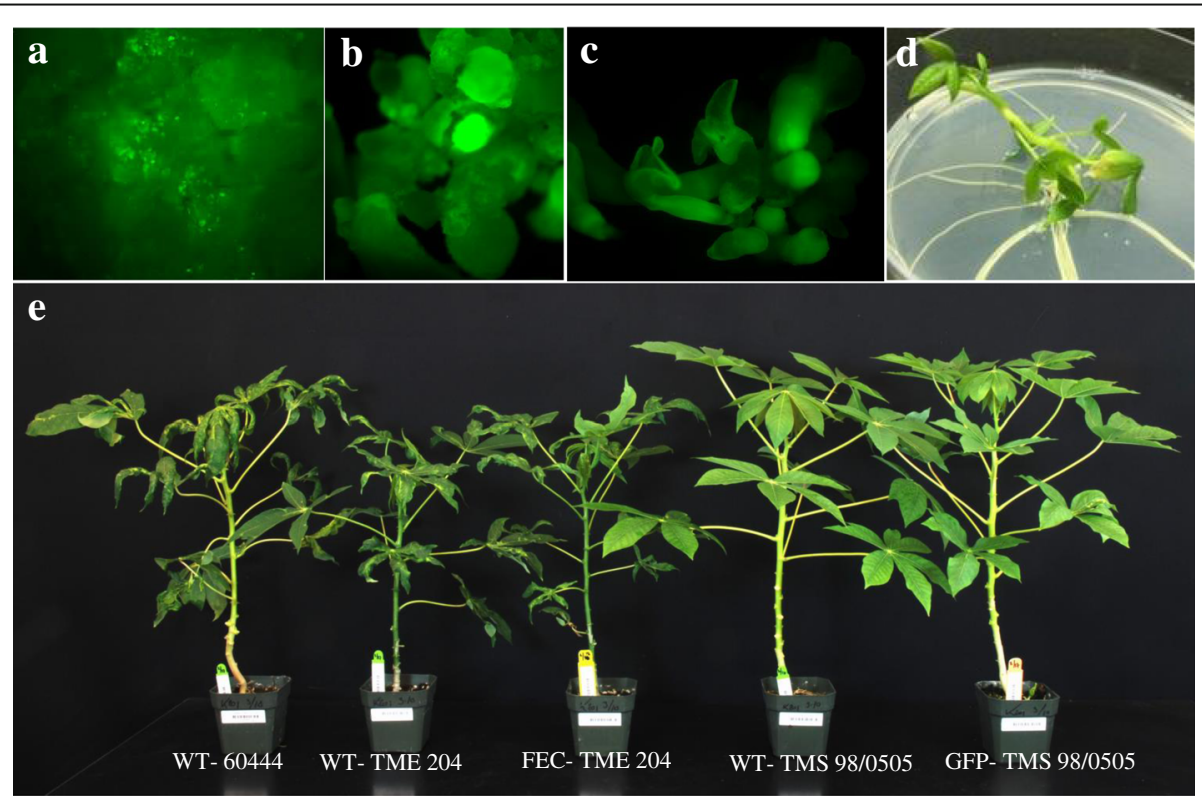

Fig. 2 Agrobacterium-mediated genetic transformation of TMS 98/0505 and response of transgenic plants to inoculation with the infectious geminivirus clone EACMV-K201. a transient GFP expression after 4 days co-culture with A. tumefaciens. b GFP-expressing callus line. c GFP-expressing somatic embryos on regeneration media. $\mathbf{d}$ Transgenic rooted plant. e Response of transgenic and micropropagated wild-type plants to EACMV-K201 at 33 days post inoculation 

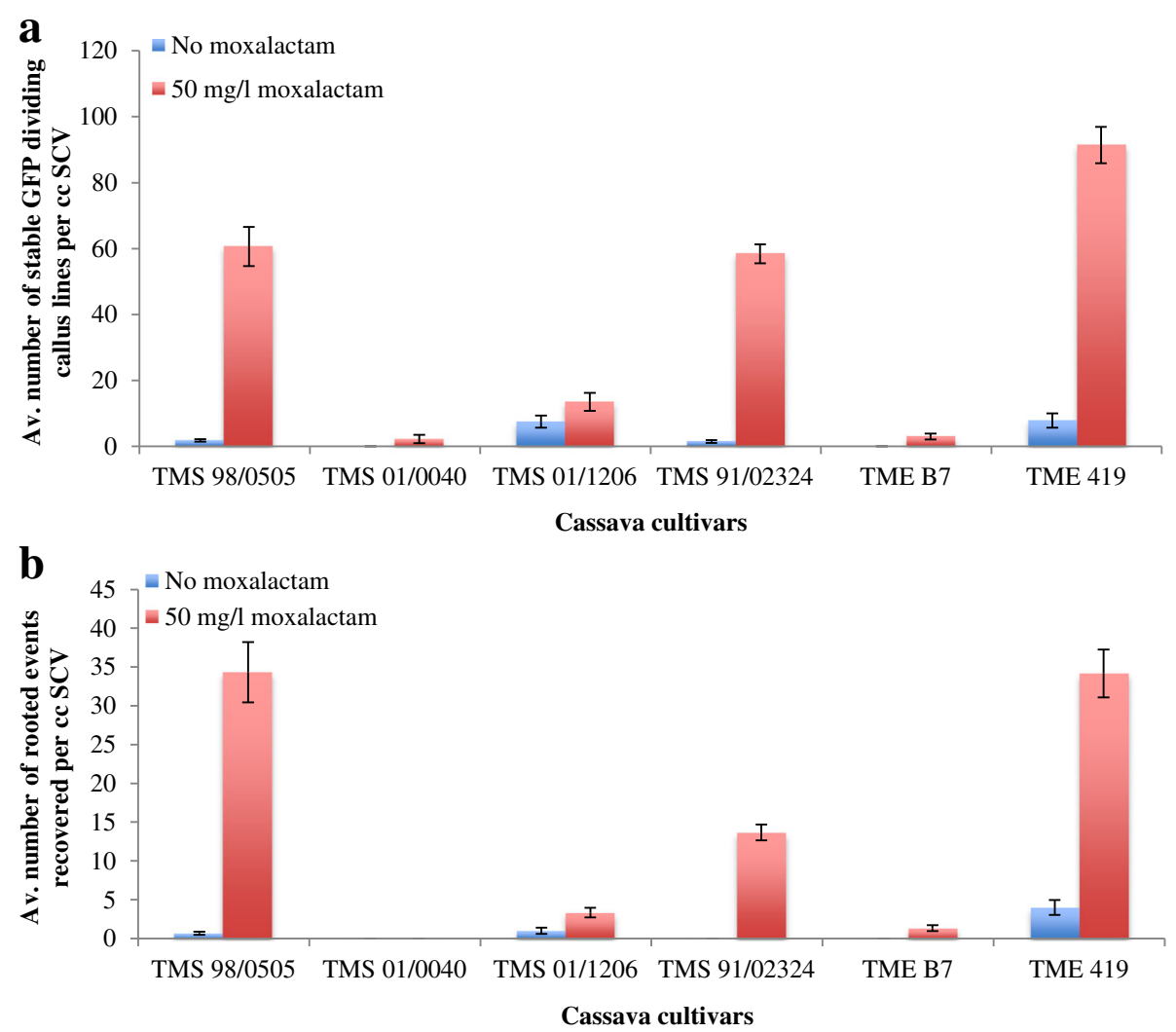

Fig. 3 Stable GFP-expressing transgenic events recovered from friable embryogenic callus (FEC) of different cassava cultivars. a Average number of GFP positive callus lines obtained after 5 weeks of co-culture. $\mathbf{b}$ Average number of GFP positive rooted events obtained after 4-5 months of co-culture. Values are Average $\pm \mathrm{SE}$, Number of experiments done $=2$ and Replications $=3$ per experiment

somatic embryogenesis (OES and FEC), genetic transformation and plant regeneration (Fig. 2e).

\section{Effect of organogenesis and meristem tip culture on CMD resistance}

We recently described a novel regeneration system in cassava by which plants are recovered from different explant types via caulogenesis. Explants are first cultured on medium containing $1 \mu \mathrm{M} 2,4-\mathrm{D}$ and $1 \mu \mathrm{M} m \mathrm{~T}$ for 7 days, followed by subculture onto medium supplemented with $6 \mu \mathrm{M} m \mathrm{~T}$ [11]. Shoots that regenerate on the second-stage medium originate from a hard, dark green colored callus, with no evidence for the occurrence of somatic embryogenesis. Plants of CMD2-type cultivars TME 204 and TME 7 were regenerated from leaf-petiole explants cultured on $m \mathrm{~T}$ [11], established in the greenhouse and inoculated with MeSPY1-VIGS and EACMV-K201 to determine if they had retained resistance to CMD. Loss of resistance to CMD occurred in both cultivars, but only from a portion of the regenerated plant lines. In TME 7, six out of 22 plant lines regenerated through caulogenesis had lost resistance to CMD (Fig. 5a \& b; Table 2). Of 11 independent TME
204 regenerant lines inoculated with MeSPY1-VIGS, seven lines were found to have retained resistance, and four to have become susceptible to CMD (Fig. $5 \mathrm{c} \& \mathrm{~d}$; Table 2). All clonal replicates derived from a given regenerated plant line behaved in the same manner, whether resistant or susceptible. When challenged with the EACMV infectious clone ECAMV-K201, the same plant lines from both cultivars remained resistant or susceptible as assessed by their ability to recover from CMD symptoms (Additional file 1: Figure S1).

Meristem tip culture is a well-established method for recovering pathogen-free plants in cassava and many other plant species [22]. The CMD2-type cultivars TME 204, TME 7 and TME 14, the CMD1-type cultivar TMS 30752 and CMD3-type TMS 98/0505 were subjected to meristem tip culture to determine effects of this tissue culture system on CMD resistance (Table 3). Maximum plant regeneration was observed in TME 14 followed by TME 7 and TMS 98/0505. TME 204 showed the lowest shoot regeneration rate with only $12 \%$ explants inducing shoots. When inoculated with MeSPY1-VIGS, two out of 17 regenerated plant lines in TME 7 and one out of 19 regenerants in TME 14 were found to have become 

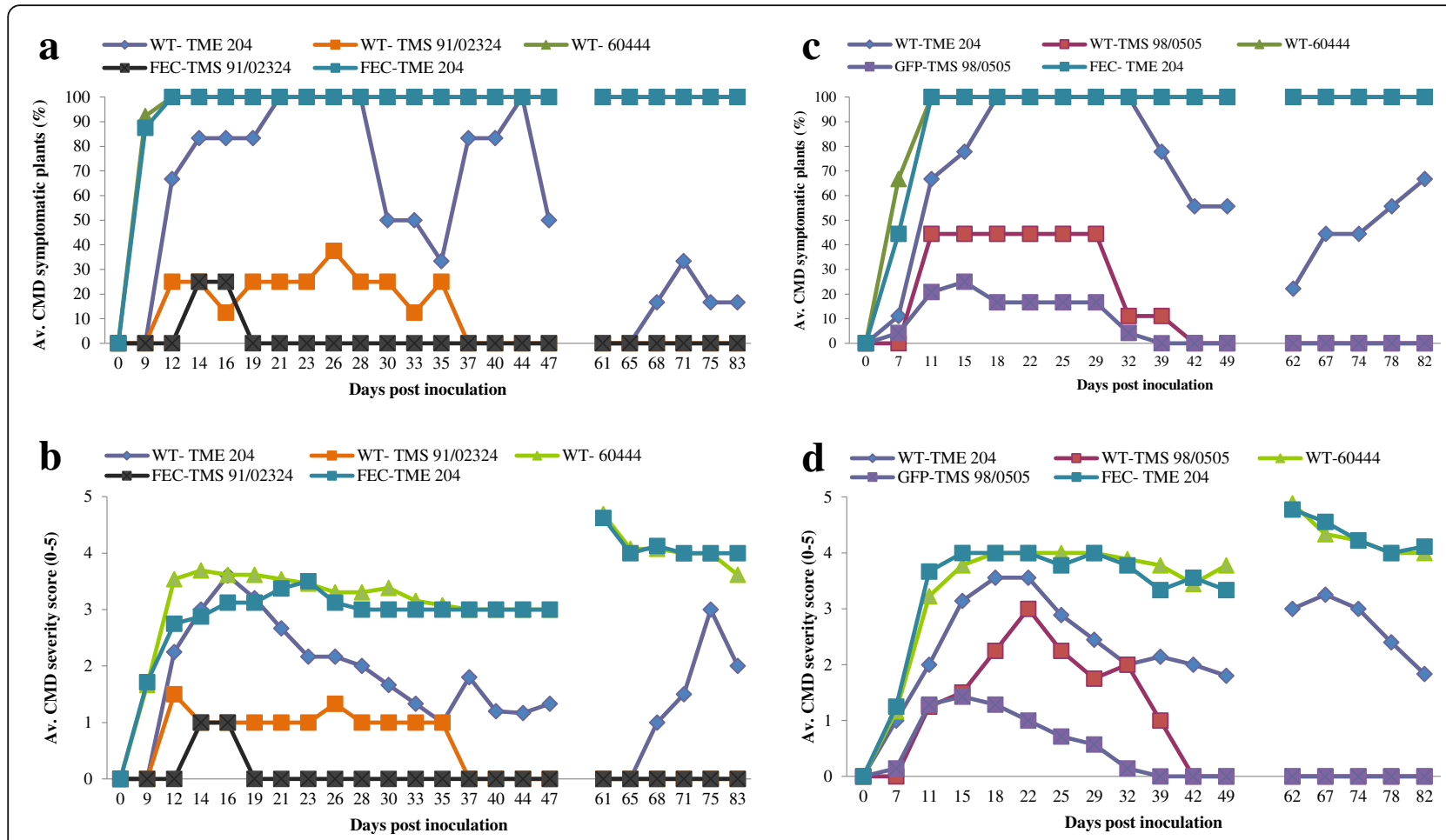

Fig. 4 Response of non-transgenic and transgenic cassava plants to inoculation with the infectious geminivirus clone EACMV-K201. Nontransgenic and transgenic plants of TMS 91/02324 and CMD3-type cultivar TMS 98/0505, respectively, were generated from FEC. a Percentage of cassava mosaic disease (CMD) symptomatic plants of FEC-derived and micropropagated TMS 91/02324. b Average CMD symptom severity scores (scale 0-5) on FEC-derived and micropropagated TMS 91/02324. c Percentage of CMD symptomatic plants of transgenic GFP expressing TMS 98/ 0505 and wild-type TMS 98/0505. d Average CMD symptom severity scores (scale 0-5) on GFP-expressing TMS 98/0505 and wild-type TMS 98/ 0505. Plant stems were cut back 48 days after biolistic inoculation and CMD assessed on new leaf growth. Breaks in the $x$ axis indicate a lapse in shoot regrowth after stem cut-back

susceptible to CMD. The remaining plant lines in these and the other cultivars tested retained resistance to CMD, recovering to establish healthy plants (Table 3, Fig. 6). Similar results were obtained when the selected meristem tip-derived plants were challenged with the relatively mild infectious clone ACMV-CM (Fig. 7).

\section{Discussion}

Morphogenic culture systems are central to the production of transgenic cassava plants and are being adapted for gene editing applications $[8,9]$. In many cases, the intention is to deploy the resulting enhanced materials to farmers and/or breeders. Compromised resistance to CMD within such plant lines is therefore a significant concern. Beyene et al. [4] reported that cassava cultivars possessing the dominant, monolocus CMD2-type resistance lost resistance to CMD when passed through somatic embryogenesis. It is essential that full understanding of the developmental and molecular mechanisms underlying loss of resistance to CMD is elucidated. This is required to secure long-term confidence in cassava plants regenerated through tissue culture, to enable improvement of CMD2-type cultivars through genetic engineering and gene-editing technologies, and to understand if and how morphogenic systems could also result in loss of critical traits in other crops. The objectives of the present study were to increase understanding of this phenomenon by screening a wider population of West African elite cassava cultivars for resistance to CMD after somatic embryogenesis, and to determine if alternative morphogenic systems also result in loss of CMD resistance.

Table 2 Response of organogenesis-derived plants to MeSPY1-VIGS challenge

\begin{tabular}{lll}
\hline Cultivar name & No. of dead independent regenerants/total regenerants challenged with MeSPY1-VIGS & Percentage CMD susceptible plants \\
\hline TME 7 & $6 / 22$ & 27 \\
TME 204 & $4 / 11$ & 36 \\
\hline
\end{tabular}




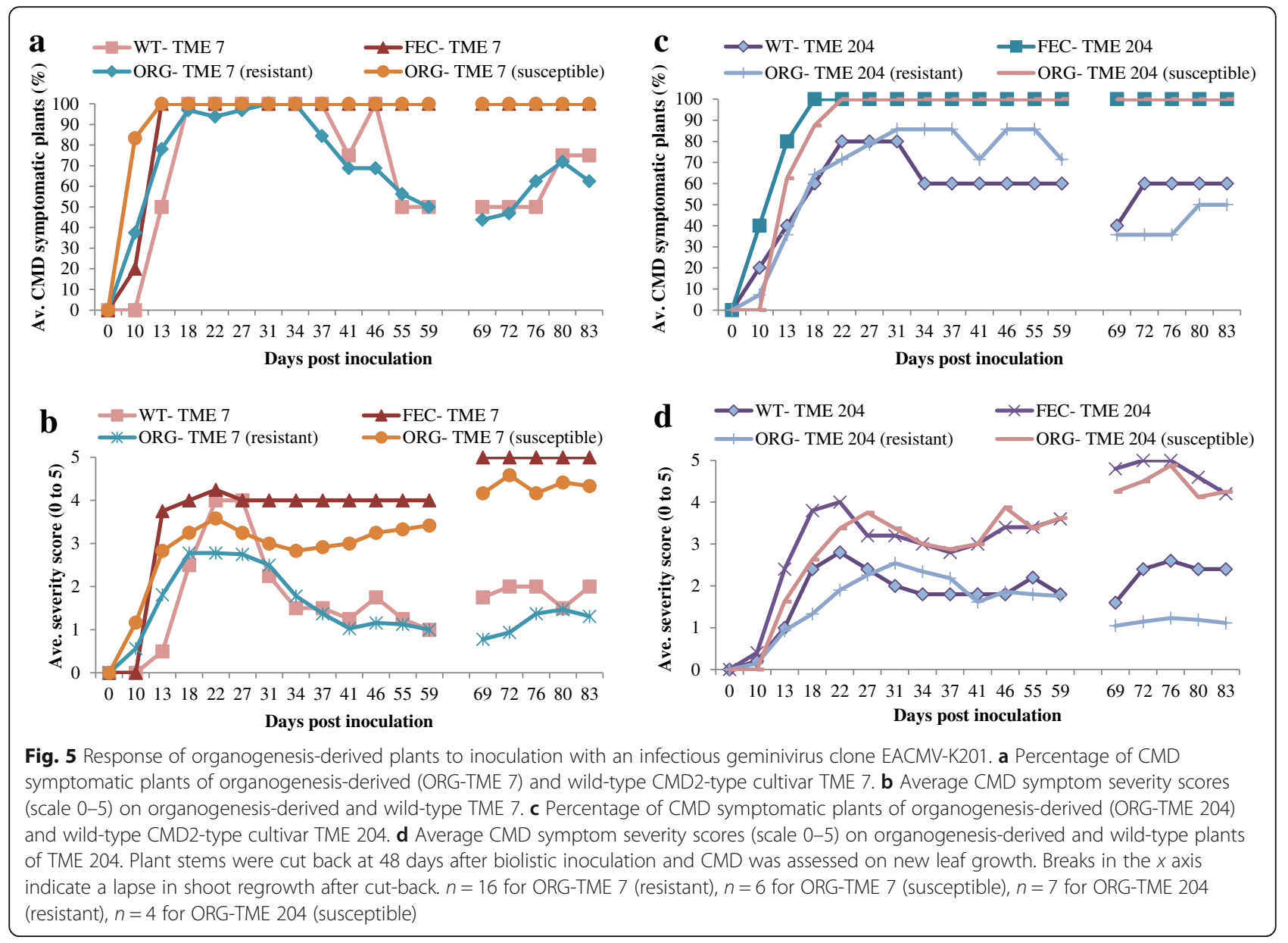

Twenty-one cassava cultivars were passed through somatic embryogenesis and subjected to CMD challenge under greenhouse conditions. While CMD2-type cassava became susceptible in the manner reported by Beyene, et al. [4], 15 elite cassava cultivars were confirmed to retain resistance to CMD when regenerated from somatic embryos. FEC produced from TMS 98/0505, TMS 01/ 0040, TMS 01/0126 and TMS 91/02324 was found to be amenable to Agrobacterium-mediated transformation. In all cases, use of moxolactam significantly enhanced production of transgenic tissues and plants. Robust resistance, equivalent to that of the non-modified wild-type plants, was demonstrated in cultivars TMS 98/0505 and TMS 91/02324 after regeneration from all stages of somatic embryogenesis and in transgenic plants of TMS 98/ 0505. High confidence can be placed, therefore, on the use of existing somatic embryogenesis protocols to introduce desirable traits through transgenic or gene editing technologies in these cultivars.

CMD2-type cultivars are widely grown by farmers in East, West and Central Africa and employed in breeding programs $[2,6,23]$. There is desire to apply biotechnology to improve these varieties for traits including resistance to CBSD [24-26], nutritional enhancement [27, 28]

Table 3 Response of meristem tip-derived plants to inoculation with MeSPY1 -VIGS challenge

\begin{tabular}{llllll}
\hline Cultivar name & $\begin{array}{l}\text { No. of explants } \\
\text { (meristem tip) } \\
\text { established }\end{array}$ & $\begin{array}{l}\text { No. of explants } \\
\text { forming shoots }\end{array}$ & $\begin{array}{l}\text { Percentage shoot } \\
\text { regeneration }\end{array}$ & $\begin{array}{l}\text { No. of dead independent } \\
\text { regenerants/total regenerants } \\
\text { challenged with MeSPY1-VIGS }\end{array}$ & $\begin{array}{l}\text { Percentage CMD } \\
\text { susceptible plants }\end{array}$ \\
\hline TME 7 & 58 & 28 & 48 & $2 / 17$ & 12 \\
TME 14 & 40 & 20 & 50 & $1 / 19$ & 5 \\
TME 204 & 58 & 7 & 12 & $0 / 1$ & 0 \\
TMS 30572 & 18 & 8 & 44 & $0 / 3$ & 0 \\
TMS 98/0505 & 58 & 13 & 22 & $0 / 3$ & 0 \\
\hline
\end{tabular}

${ }^{\mathrm{a}}$ Explants were setup in two separate experiments 


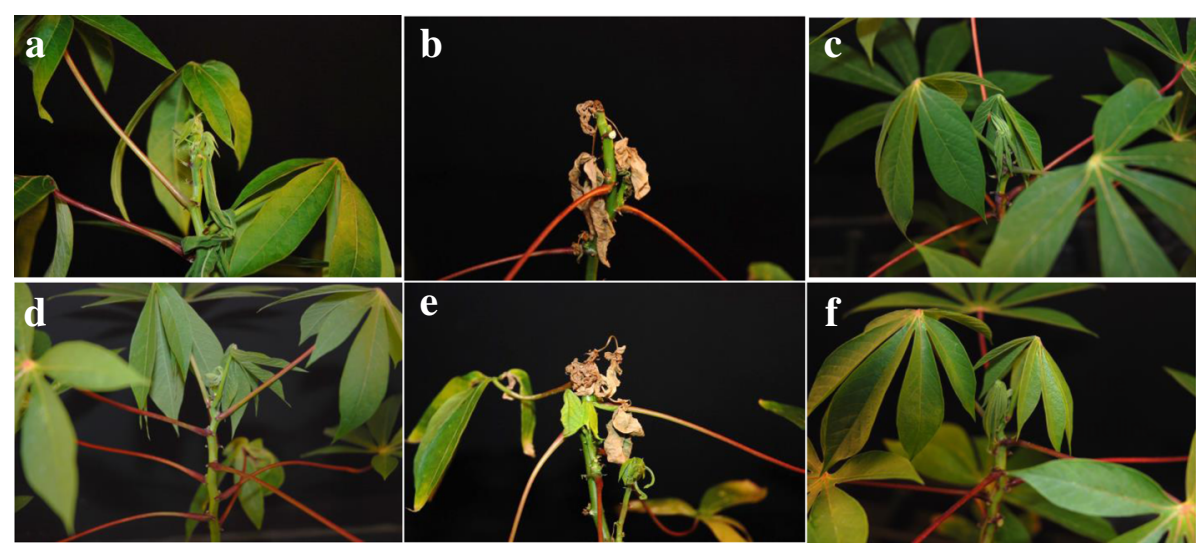

Fig. 6 Response of meristem tip culture-derived plants of TME 7, TME 14, TME 7, TMS 98/0505 to inoculation with MeSPY1-VIGS. Silencing of MeSPY using MeSPY1-VIGS leads to shoot-tip necrosis and death of the of CMD susceptible cassava plants within 2-4 weeks. a CMD resistant micropropagated TME 7 plant. b CMD susceptible meristem tip-derived TME 7 plant. c CMD resistant meristem tip-derived TME 7 plant. d CMD resistant wild-type TME 14 plant. e CMD susceptible meristem tip-derived TME 14 plant. f CMD resistant meristem tip-derived TME 14 plant

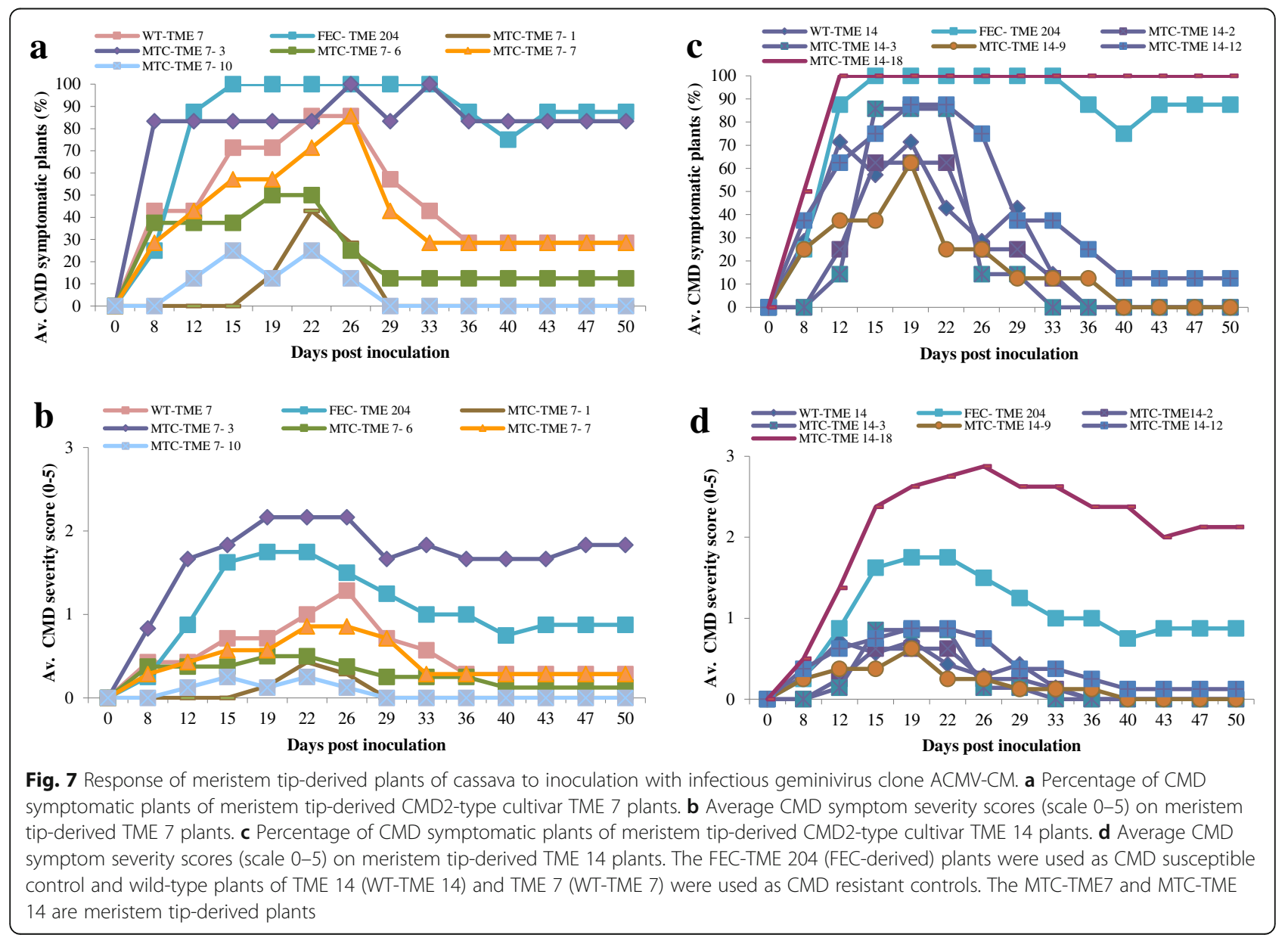


and post-harvest qualities [29] but, as stated above, this must occur without losing the critical trait for CMD resistance. Efforts to develop plant regeneration systems that circumvent the need for somatic embryogenesis resulted in the caulogenic system reported recently by Chauhan and Taylor [11]. In the present study, plants of the CMD2-type cultivars TME 7 and TME 204 regenerated though this cytokinin-based shoot regeneration process were challenged with geminiviruses. In both cultivars, a proportion of the regenerated plant lines were confirmed to have lost resistance to CMD. This response was uniform and stable across clonal replicates of a given line, such that all plants of a regenerated resistant line remained resistant, and those that were susceptible remained susceptible.

Data from plants regenerated through caulogenesis and meristem tip culture provide clear evidence that somatic embryogenesis per se is not the underlying cause for loss of resistance to geminiviruses. Unlike somatic embryogenesis, shoot regeneration using meta-topolin does not involve exposure of tissues to high levels of auxin, nor the somaclonal variation associated with such culture systems. It remains unknown how loss of resistance occurs, and why 27$36 \%$ of plant lines regenerated via caulogenesis lost resistance, while others remained fully resistant. A possible explanation is that disruption of shoot meristem integrity may be an underlying contributor to loss of CMD resistance in CMD2-type regenerated plants. To test this hypothesis, meristem tip culture was investigated in CMD2-type cultivars. In this case, a small percentage $(5-12 \%)$ of plant lines regenerated from both CMD2-type cultivars (TME 7 and TME 14) were found to have lost resistance to CMD. These plants were susceptible even to a relatively less virulent strain of ACMV-CM, in the same manner described previously for somatic embryo-derived plants [4]. An alternative hypothesis is that epigenetic changes occur as a result of morphogenesis in CMD2-type cassava cultivars. Such changes may affect resistance gene(s) and/or susceptibility genes at the CMD2 locus. Indeed, it has previously been shown in five cassava cultivars that the meristem-derived plants were epigenetically different than the field grown plants [5]. Additional studies are underway to test these hypotheses.

\section{Conclusions}

The information presented here has important implications for biotechnological applications in cassava, and efforts to elucidate mechanisms of resistance to CMD. Non-CMD2-type cultivars are not affected by passage through somatic embryogenesis or other morphogenic systems, and can therefore be used with confidence as targets for transgenic and gene editing enhancement and mass propagation through tissue culture. Secondly, regeneration via caulogenesis provides a potential solution for generating modified CMD2-type cultivars that retain resistance to $\mathrm{CMD}$. However, plants regenerated in this manner require testing for their resistance to geminiviruses to eliminate those that have been compromised. Finally, meristem tip culture should be used with caution when applied to cassava cultivars carrying the CMD2-type mechanism because it is possible to lose resistance to CMD in plants recovered through this regeneration system. As for caulogenesis, regenerated plant lines should be tested empirically to confirm that CMD resistance is fully functional before dissemination to farmers or establishment in germplasm collections. Loss of resistance through three culture systems provides a powerful toolset for investigating the molecular mechanism behind CMD resistance. The information described here will be critical for designing experiments and interpreting genomic, transcriptomic and epigenomic datasets focused on such efforts.

\section{Additional file}

\begin{abstract}
Additional file 1: Figure S1. Response of organogenesis-derived plants of cassava to inoculation with an infectious geminivirus clone of EACMVK201 and MeSPY1-VIGS. a EACMV-K201 (left) and MeSPY1-VIGS (right) challenged plants of micropropagated TME 7. b EACMV-K201 (left) and MeSPY1-VIGS (right) challenged FEC-derived plants of TME 7. c \& d EACMV-K201 (left) and MeSPY1-VIGS (right) challenged organogenesisderived plants of TME 7. e EACMV-K201 (left) and MeSPY1-VIGS (right) challenged plants of micropropagated TME 204. f EACMV-K201 (left) and MeSPY1-VIGS (right) challenged FEC-derived plants of TME 204. g \& h EACMV-K201 (left) and MeSPY1-VIGS (right) challenged organogenesisderived plants of TME 204. (PPTX $72459 \mathrm{~kb}$ )
\end{abstract}

Acknowledgements

We thank the technical assistance provided by Jenny Tran, Stephanie Lamb, Danielle Stretch, Jennifer Winch, Claire Albin, Collin Leubbert, Paula Butts, Jackson Gehan and Theodore Moll.

\section{Funding}

This work was supported by the Bill and Melinda Gates Foundation. The funding body had no role in the design of the study; collection, analysis, and interpretation of data; or in writing the manuscript.

\section{Availability of data and materials}

All data generated and analysed during this study are included in this published article and its Additional file 1: Figure S1.

\section{Authors' contributions}

RC and NT conceived the experiments. RC, GB and NT designed the experiments. RC and GB executed the experiments. RC and NT wrote the manuscript. All authors read and approved the manuscript.

Ethics approval and consent to participate Not applicable.

Consent for publication

Not applicable.

Competing interests

The authors declare that they have no competing interests. 


\section{Publisher's Note}

Springer Nature remains neutral with regard to jurisdictional claims in published maps and institutional affiliations.

Received: 15 December 2017 Accepted: 17 June 2018

Published online: 25 June 2018

\section{References}

1. IITA. Annual report (International Institute of Tropical Agriculture). Ibadan: IITA; 1990.

2. Okogbenin E, Moreno I, Tomkins J, Fauquet CM, Mkamilo G, Fregene M. Marker-assisted breeding for cassava mosaic disease resistance. In: Varshney R, Tuberosa R, editors. Translational genomics for crop breeding: biotic stress, Volume 1. Chichester: John Wiley \& Sons Ltd 2013. p. 291-325

3. Okogbenin E, Egesi CN, Olasanmi B, Ogundapo O, Kahya S, Hurtado P, et al. Molecular marker analysis and validation of resistance to cassava mosaic disease in elite cassava genotypes in Nigeria. Crop Sci. 2012;52:2576-86.

4. Beyene G, Chauhan RD, Wagaba H, Moll T, Alicai T, Miano D, Carrington J, Taylor NJ. Loss of CMD2-mediated resistance to cassava mosaic disease in plants regenerated through somatic embryogenesis. Mol Plant Pathol. 2016; 17:1095-110.

5. Kitimu SR, Taylor J, March TJ, Tairo F, Wilkinson MJ, Rodriguez Lopez CM. Meristem micropropagation of cassava (Manihot esculenta) evokes genome-wide changes in DNA methylation. Front Plant Sci. 2015;6:590.

6. Rabbi IY, Hamblin MT, Kumar PL, Gedil MA, Ikpan AS, Jannink J-L, Kulakow PA. High-resolution mapping of resistance to cassava mosaic geminiviruses in cassava using genotyping-by-sequencing and its implications for breeding. Virus Res. 2014;186:87-96.

7. Ndunguru J, De Leon L, Doyle CD, Sseruwagi P, Plata G, Legg JP, et al. Two novel DNAs that enhance symptoms and overcome CMD2 resistance to cassava mosaic disease. J Virol. 2016;90:4160-73.

8. Odipio J, Alicai T, Ingelbrecht I, Nusinow DA, Bart R, Taylor NJ. Efficient CRISPR/Cas9 genome editing of phytoene desaturase in cassava. Front Plant Sci. 2017:8:1780.

9. Hummel A, Chauhan R, Cermak T, Mutka A, Vijayaraghavan A, Boyher A, et al. Allele exchange at the EPSPS locus confers glyphosate tolerance in cassava. Plant Biotechnol J. 2017; https://doi.org/10.1111/pbi.12868. [Epub ahead of print].

10. Chauhan RD, Beyene G, Kalyaeva M, Fauquet CM, Taylor N. Improvements in agrobacterium-mediated transformation of cassava (Manihot esculenta Crantz) for large-scale production of transgenic plants. Plant Cell Tissue Organ Cult. 2015;121:591-603.

11. Chauhan RD, Taylor NJ. Meta-topolin stimulates de novo shoot organogenesis and plant regeneration in cassava. Plant Cell Tissue Organ Cult. 2018;132:219-24.

12. IITA. Cassava in vitro processing and gene Banking. Ibadan: IITA. https:// cropgenebank.sgrp.cgiar.org/images/file/learning_space/iita_Cassava_ genebank_manual.pdf; 2007.

13. Taylor N, Gaitan-Solis E, Moll T, Trauterman B, Jones T, Pranjal A, et al. A high-throughput platform for the production and analysis of transgenic cassava (Manihot esculenta) plants. Trop Plant Biol. 2012;5:127-39.

14. Driver J, Kuniyuki A. In vitro propagation of paradox walnut rootstock. Hortic Sci. 1984:19:507-9.

15. Murashige T, Skoog F. A revised medium for rapid growth and bio assays with tobacco tissue cultures. Physiol Plant. 1962:15:473-97.

16. Beyene $\mathrm{G}$, Chauhan RD, Taylor NJ. A rapid virus-induced gene silencing (VIGS) method for assessing resistance and susceptibility to cassava mosaic disease. Virol J. 2017b;14:47.

17. Bull SE, Briddon RW, Sserubombwe WS, Ngugi K, Markham PG, Stanley J. Genetic diversity and phylogeography of cassava mosaic viruses in Kenya. J Gen Virol. 2006;87:3053-65.

18. Patil BL, Fauquet CM. Differential interaction between cassava mosaic geminiviruses and geminivirus satellites. J Gen Virol. 2010;91:1871-82.

19. Fondong VN, Pita JS, Rey ME, de Kochko A, Beachy RN, Fauquet CM. Evidence of synergism between African cassava mosaic virus and a new double-recombinant geminivirus infecting cassava in Cameroon. J Gen Virol. 2000;81:287-97.

20. Fauquet C, Fargette D. African cassava mosaic virus: etiology, epidemiology and control. Plant Dis. 1990;74:404-11.
21. Gresshoff PM, Doy $\mathrm{CH}$. Derivation of a haploid cell line from Vitis vinifera and the importance of the stage of meiotic development of the anthers for haploid culture of this and other genera. Zt Pflanzenphysiol. 1974;73:132-41.

22. Grout BWW. Meristem-tip culture for propagation and virus elimination. In: Hall RD, editor. Methods in molecular biology, plant cell culture protocols, vol. 111. Totowa: Humana Press; 1999. p. 115-25.

23. Kawuki R, Pariyo A, Amuge T, Nuwamanya E, Ssemakula G, Tumwesigye S, et al. A breeding scheme for local adoption of cassava (Manihot esculenta Crantz). J Plant Breed Crop Sci. 2011;3:120-30.

24. Patil BL, Legg JP, Kanju E, Fauquet CM. Cassava brown streak disease: a threat to food security in Africa. J Gen Virol. 2015;96:956-68.

25. Wagaba H, Beyene G, Aleu J, Odipio J, Okao-Okuja G, Chauhan RD, et al. Field level RNAi-mediated resistance to cassava Brown streak disease across multiple cropping cycles and diverse east African agro-ecological locations. Front Plant Sci. 2016:7:2060.

26. Beyene G, Chauhan RD, Ilyas M, Wagaba H, Fauquet CM, Miano D, Alicai T, Taylor NJ. A virus-derived stacked RNAi construct confers robust resistance to cassava Brown streak disease. Front Plant Sci. 2017a;7:2052.

27. Adenle A, Aworh O, Akromah R, Parayil G. Developing GM super cassava for improved health and food security: future challenges in Africa. Agric Food Secur. 2012;1:11

28. Narayanan N, Beyene G, Chauhan RD, Gaitan-Solis E, Grusak MA, Taylor N, Anderson P. Overexpression of Arabidopsis VIT1 increases accumulation of iron in cassava roots and stems. Plant Sci. 2015:240:170-81.

29. Zainuddin IM, Fathoni A, Sudarmonowati E, Beeching JR, Gruissem W, Vanderschuren $\mathrm{H}$. Cassava post-harvest physiological deterioration: from triggers to symptoms. Postharvest Biol Technol. 2018;142:115-23.

\section{Ready to submit your research? Choose BMC and benefit from:}

- fast, convenient online submission

- thorough peer review by experienced researchers in your field

- rapid publication on acceptance

- support for research data, including large and complex data types

- gold Open Access which fosters wider collaboration and increased citations

- maximum visibility for your research: over $100 \mathrm{M}$ website views per year

At BMC, research is always in progress.

Learn more biomedcentral.com/submissions 